\title{
The seroepidemiology of pertussis in NSW: fluctuating immunity profiles related to changes in vaccination schedules
}

\author{
Helen E. Quinn ${ }^{\mathrm{A}, \mathrm{E}}$, Deepika Mahajan ${ }^{\mathrm{A}}$, \\ Linda Hueston $^{\mathrm{B}}$, Patricia Campbell ${ }^{\mathrm{C}, \mathrm{D}}$, \\ Robert I. Menzies ${ }^{\mathrm{A}}$, Gwendolyn L. Gilbert ${ }^{\mathrm{B}}$ \\ and Peter B. McIntyre ${ }^{\mathrm{A}}$ \\ ${ }^{\mathrm{A}}$ National Centre for Immunisation Research and Surveillance, \\ The Children's Hospital at Westmead \\ ${ }^{\mathrm{B}}$ Centre for Infectious Diseases and Microbiology - Public Health, \\ Institute for Clinical Pathology and Medical Research, \\ Westmead Hospital \\ ${ }^{\mathrm{C}}$ Vaccine and Immunisation Research Group, Murdoch Children's \\ Research Institute \\ Delbourne School of Population Health, The University \\ of Melbourne \\ ${ }^{\mathrm{E}}$ Corresponding author.Email: HelenQ@chw.edu.au
}

\begin{abstract}
The pertussis epidemic experienced in NSW in 2008-2009 was likely to be in part due to changes in diagnostic practice since 2007, which amplified disease notifications. We used populationbased seroepidemiology as a less biased means of interpreting age-specific pertussis infection patterns in NSW from three serosurveys undertaken in 1997-98 (during an epidemic), 2002 (post-epidemic) and 2007 (inter-epidemic), using a standardised pertussis toxin IgG enzyme-linked immunosorbent assay (ELISA). There was a decrease in the proportion of high anti-pertussis toxin IgG titres $(>62.5$ ELISA Units $/ \mathrm{mL}$ ) across all age groups in the 2007 serosurvey compared to the previous two serosurveys. In the 2007 serosurvey, the proportion of undetectable $(<5$ ELISA Units $/ \mathrm{mL})$ anti-pertussis toxin IgG titres increased in many age groups. The seroepidemiological profiles of the three serosurveys demonstrate fluctuating immunity profiles related to changes in vaccination schedules.
\end{abstract}

on a background of endemic circulation. In 2008-2009, New South Wales (NSW) experienced a sustained pertussis epidemic which was unusually large in magnitude. ${ }^{1}$ However, interpretation of notification data from the current pertussis epidemic is confounded by changes in diagnostic practice since 2007. The shift to widespread use of polymerase chain reaction for pertussis diagnosis in all age groups is likely to have amplified the number of cases notified during the epidemic, particularly in children.

Population-based, cross-sectional seroepidemiology offers a less biased means of comparison of age-specific patterns of pertussis compared to other disease surveillance methods, but requires acceptable standardisation and reproducibility of serologic tests and the ability to extrapolate these results to estimates of symptomatic cases. The European Sero-Epidemiology Network (ESEN) standardised the use of a serologic criterion for recent infection, measured in IgG-pertussis toxin (PT) using enzyme-linked immunosorbent assay (ELISA) Units $^{2}$ (EU) between participating laboratories using different serologic methods. This development allowed meaningful comparison of Bordetella pertussis seroepidemiology in six European countries. ${ }^{3,4}$ This standardised methodology has previously been applied to Australian sera collected in 1997-98 and 2002 to describe national pertussis trends by age and over time. ${ }^{5,6}$ Since then, several important changes in pertussis vaccine schedules have occurred in all states and territories, including NSW. First, the 18-month booster dose of diphtheriatetanus-acellular pertussis vaccine (DTPa) was removed from the National Immunisation Program schedule in 2003. Second, an adult formulation acellular pertussis-containing vaccine (dTpa) was added to the schedule for adolescents, with school-based vaccination commencing in May $2004 .^{7}$ Earlier schedule changes are outlined in Table 1.

This study compares NSW data from three cross-sectional serosurveys, undertaken in 1997-98 (during an epidemic), 2002 (post-epidemic) and 2007 (inter-epidemic). The aim was to evaluate age-specific patterns of presumptive recent pertussis infection in the context of changes to the vaccine schedule and pertussis notifications.

\section{Methods}

Population and study design

The 2007 sera used in this study were selected from a bank of approximately 7200 sera collected opportunistically 
Table 1. Significant pertussis vaccine schedule changes in NSW, 1975-2003

\begin{tabular}{|c|c|c|}
\hline Year & Vaccine type & Event \\
\hline \multirow[t]{2}{*}{1975} & DTPw & $\begin{array}{l}\text { Diphtheria-tetanus-whole cell pertussis (DTPw) national vaccination schedule recommended } \\
\text { for infants aged 3, } 4 \text { and } 5 \text { months }\end{array}$ \\
\hline & DTPw & Booster dose for infants aged $15-18$ months introduced \\
\hline 1978 & DTPw & Booster dose removed from schedule \\
\hline 1982 & DTPw & National vaccination schedule changed to a primary series at 2,4 and 6 months of age \\
\hline 1985 & DTPw & $\begin{array}{l}\text { Booster dose re-introduced at } 18 \text { months of age due to an increase in pertussis incidence } \\
\text { in children aged } 4-5 \text { years }\end{array}$ \\
\hline 1994 & DTPw & Booster dose at $4-5$ years of age added to the recommend vaccination schedule \\
\hline 1997 & DTPa & $\begin{array}{l}\text { Diphtheria-tetanus-acellular pertussis (DTPa) recommended for the booster doses } \\
\text { of vaccination }\end{array}$ \\
\hline 1999 & DTPa & All five scheduled doses of DTPw replaced with DTPa \\
\hline 2000 & DTPa & Second booster dose recommended at 4 years instead of $4-5$ years \\
\hline \multirow[t]{2}{*}{2003} & DTPa & Booster dose at 18 months of age removed from schedule \\
\hline & dTpa & $\begin{array}{l}\text { Adult formulated diphtheria-tetanus-acellular pertussis (dTpa) recommended as a booster } \\
\text { dose at 15-17 years of age }\end{array}$ \\
\hline
\end{tabular}

from a geographically representative group of 29 diagnostic laboratories receiving samples from hospitalised and ambulant persons throughout Australia as part of a national serosurveillance program, ${ }^{8}$ but was restricted to NSW laboratories for this study. The sera in the opportunistic sample were residual from specimens submitted for diagnostic testing and would otherwise have been discarded. Residual sera were from subjects who: were immunosuppressed; had received multiple or recent (within 3 months) blood transfusions; or were known to have HIV infection, were excluded by staff at the diagnostic laboratory. Sera were identified by a medical record number, sex, age, state/ territory of origin and a unique identifier, to ensure that only one sample from any subject was tested. Approval for the serosurvey was obtained from the Western Sydney Area Health Service Human Research Ethics Committee.

In all relevant age groups the required sample size was calculated to achieve a $95 \%$ probability of precision $\pm 7 \%$ around a point estimate based on the expected level of seroprevalence. There were equal numbers of males and females within each age group.

The population and study design for the 1997-98 and 2002 serosurveys have been described previously. ${ }^{5,6}$ Across the three serosurveys the proportion of samples from outer regional and remote locations remained similar. In contrast, the proportion of samples from a metropolitan area decreased (85\% in 1997-98 to $70 \%$ in 2007) and the proportion from an inner regional area increased (10\% in $1997-98$ to $25 \%$ in 2007 ).

Testing and serologic criteria for recent pertussis infection For the 2007 serosurvey a total of 1152 sera randomly selected from those available in each age group were tested using an established ELISA method adapted from
Giammanco et $\mathrm{al}^{9}$ and previously described in full. ${ }^{5,6}$ The 1997-98 serosurvey was conducted at the ESEN reference laboratory at the University of Palermo, Italy and both the 2002 and 2007 analyses were performed at the Centre for Infectious Diseases and Microbiology, Sydney, Australia. The method used in our laboratory was validated against a panel of sera from the ESEN reference laboratory. In 2007 the assay was again validated, using a panel of samples from the 2002 serosurvey.

The minimum level of detection of the assay, defined as the minimum amount of antibody that must be present for the serum to have at least one optical density (OD) value within the linear range of the reference serum response curve, was estimated to be $2 \mathrm{EU} / \mathrm{mL}$. Anti-PT IgG levels were divided into four categories, previously described by the ESEN study group as suggestive of pertussis exposure within certain time periods: ${ }^{4}<5 \mathrm{EU} / \mathrm{mL}$ (undetectable), $5-<62.5 \mathrm{EU} / \mathrm{mL}$ (exposure more than 12 months previously), $62.5-<125 \mathrm{EU} / \mathrm{mL}$ (exposure within 12 months) and $\geq 125 \mathrm{EU} / \mathrm{mL}$ (exposure within 6 months). Exposure implies 'significant' exposure, with antibody response and is inclusive of infection and immunisation. These categories were originally derived by de Melker and colleagues from a longitudinal cohort study of Dutch patients with clinically confirmed pertussis infection, and demonstrated that high anti-PT IgG levels can be a sensitive and specific indicator of recent infection. These levels fell below the nominated threshold levels in almost all patients within 12 months of infection. ${ }^{2}$

\section{Statistical analysis}

Proportions and 95\% confidence intervals were calculated for sera in each of the categories described above, by age group. A chi-square test was used to compare proportions by age group and serosurvey, with $p$ values $<0.05$ 


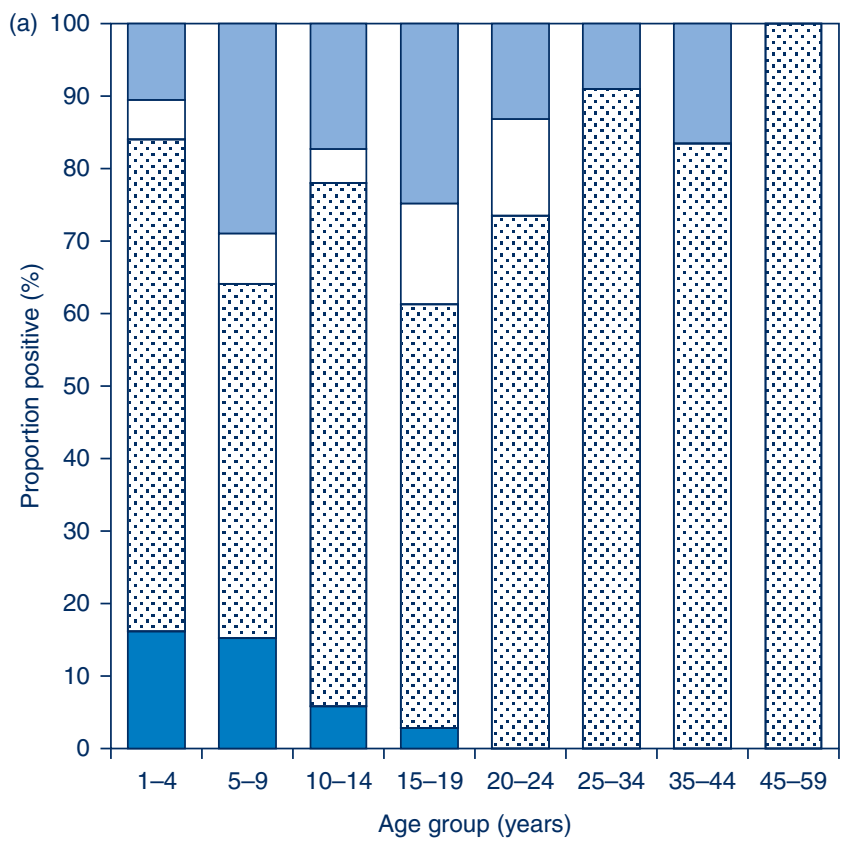

$\square \geq 125 \mathrm{EU} / \mathrm{mL} \square 62.5-<125 \mathrm{EU} / \mathrm{mL}$ ๑ 5-<2.5 EU/mL $\square<5 \mathrm{EU} / \mathrm{mL}$

Figure 1a. Cross-sectional distribution of anti-pertussis toxin IgG levels in the NSW population in eight age groups (1-4 through to 45-59 years) in 1997-98.

considered significant. Analyses were performed using Microsoft Excel and SAS (version 9.2, SAS Institute, Cary, NC, USA).

\section{Results}

The distribution of titres in each of the three serosurveys is provided in Figure 1(a,b,c). In 1997-98, the highest proportion of anti-PT IgG levels $\geq 62.5 \mathrm{EU} / \mathrm{mL}$ was seen in the 5-9 and 15-19-year age groups. In contrast, in 2002 the highest proportion of anti-PT IgG levels $\geq 62.5 \mathrm{EU} / \mathrm{mL}$ was seen in the 20-24, 25-34 and 35-44-year age groups. The proportion with anti-PT IgG levels $\geq 62.5 \mathrm{EU} / \mathrm{mL}$ was reduced across the population in 2007 compared to the previous collections.

To further highlight these differences, the proportion of sera with anti-PT IgG levels $\geq 62.5 \mathrm{EU} / \mathrm{mL}$ across the three collections are presented in Figure 2. When comparing the 2002 serosurvey to the 1997-98 serosurvey, significant decreases were seen in the 5-9 and 15-19-year age groups. When comparing the 2007 serosurvey to the 2002 serosurvey, significant decreases were seen in the 5-9 and $10-14$-year age groups and in all age groups for those aged 20 years and over.

If the population proportions with anti-PT IgG levels $<5 \mathrm{EU} / \mathrm{mL}$ (undetectable) over the period of the three collections are compared, many age groups experienced a significant increase in undetectable levels (Figure 3).

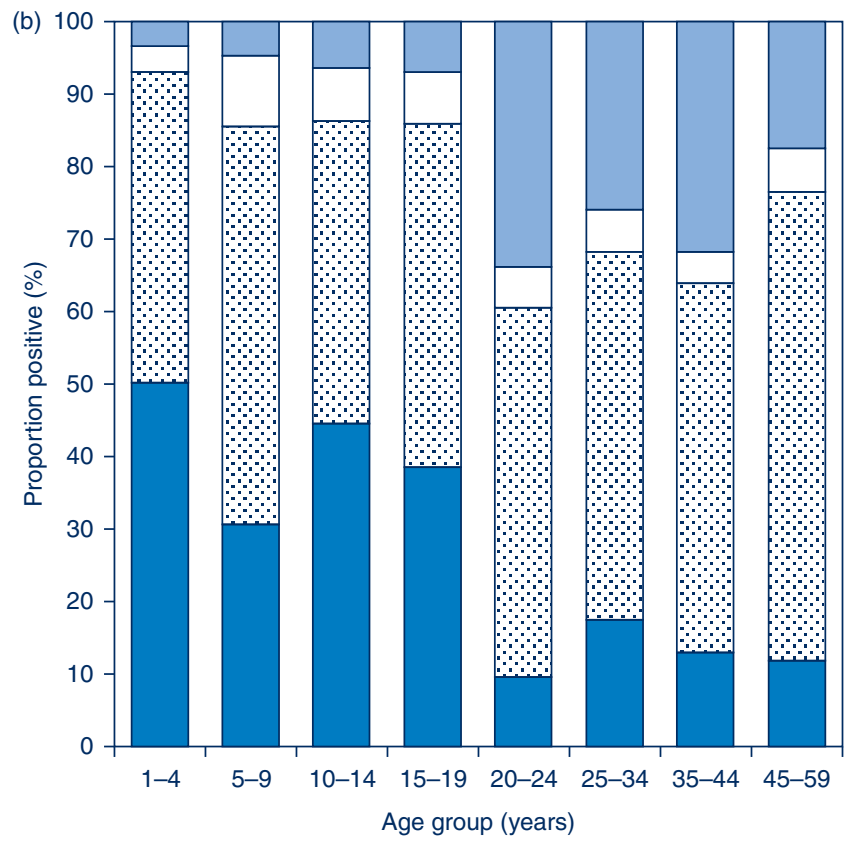

$\square \geq 125 \mathrm{EU} / \mathrm{mL} \square 62.5-<125 \mathrm{EU} / \mathrm{mL} \square 5-<62.5 \mathrm{EU} / \mathrm{mL} \square<5 \mathrm{EU} / \mathrm{mL}$

Figure $1 \mathrm{~b}$. Cross-sectional distribution of anti-pertussis toxin IgG levels in the NSW population in eight age groups (1-4 through to 45-59 years) in 2002.

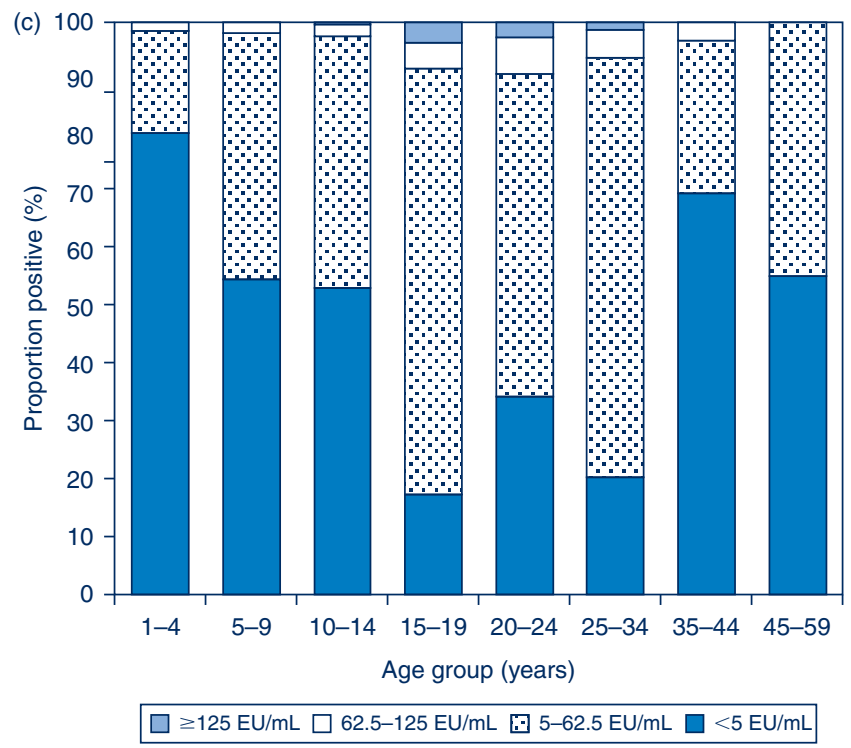

Figure 1c. Cross-sectional distribution of anti-pertussis toxin IgG levels in the NSW population in eight age groups (1-4 through to 45-59 years) in 2007.

Sources: 1997-98, 2002 and 2007 serosurveys.

In particular, the proportion of 1-4-year olds increased from $16 \%$ in $1997-98$ to $81 \%$ by 2007 and the proportion of $35-44$-year olds increased from $0 \%$ in $1997-98$ to $70 \%$ by 2007 . The only age group for which a significant decrease was observed between the 2002 and 2007 collections was 15-19-year olds. 


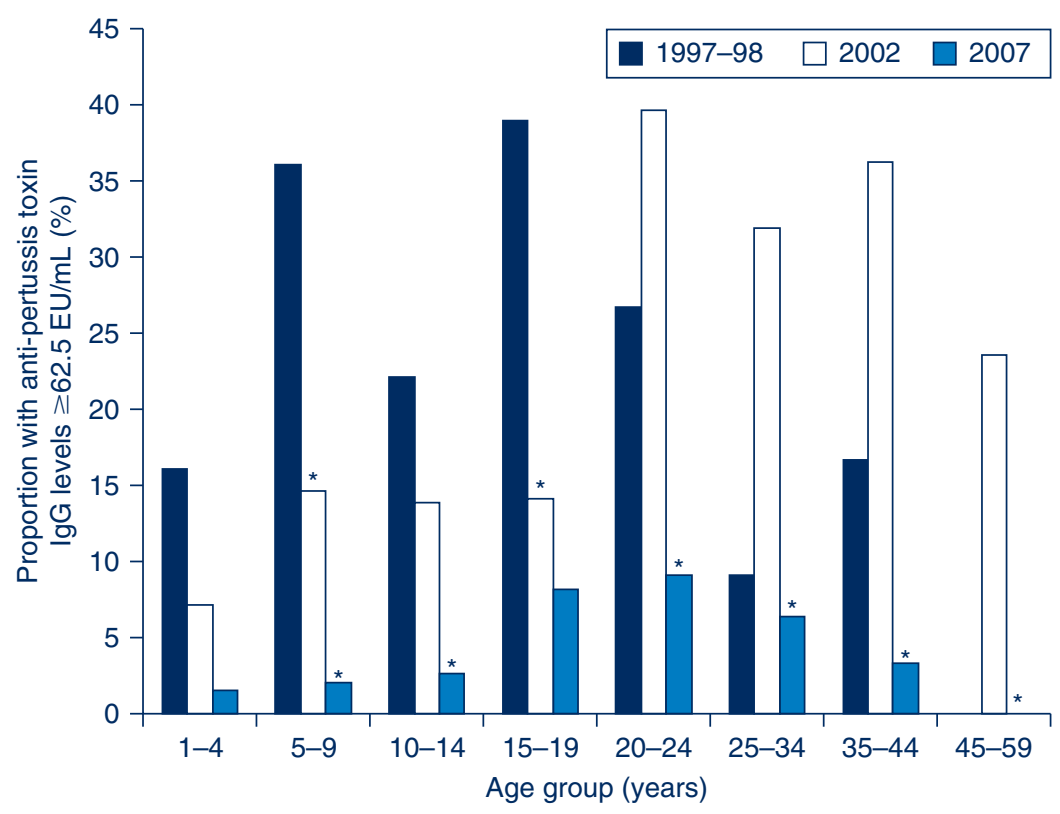

Figure 2. Cross-sectional proportion of anti-pertussis toxin IgG levels ( $\geq 62.5 \mathrm{EU} / \mathrm{mL}$ ) in the NSW population in eight age groups (1-4 years through to 45-59 years) in 1997-98, 2002 and 2007.

*Significant decreases compared to the previous collection.

Sources: 1997-98, 2002 and 2007 serosurveys.

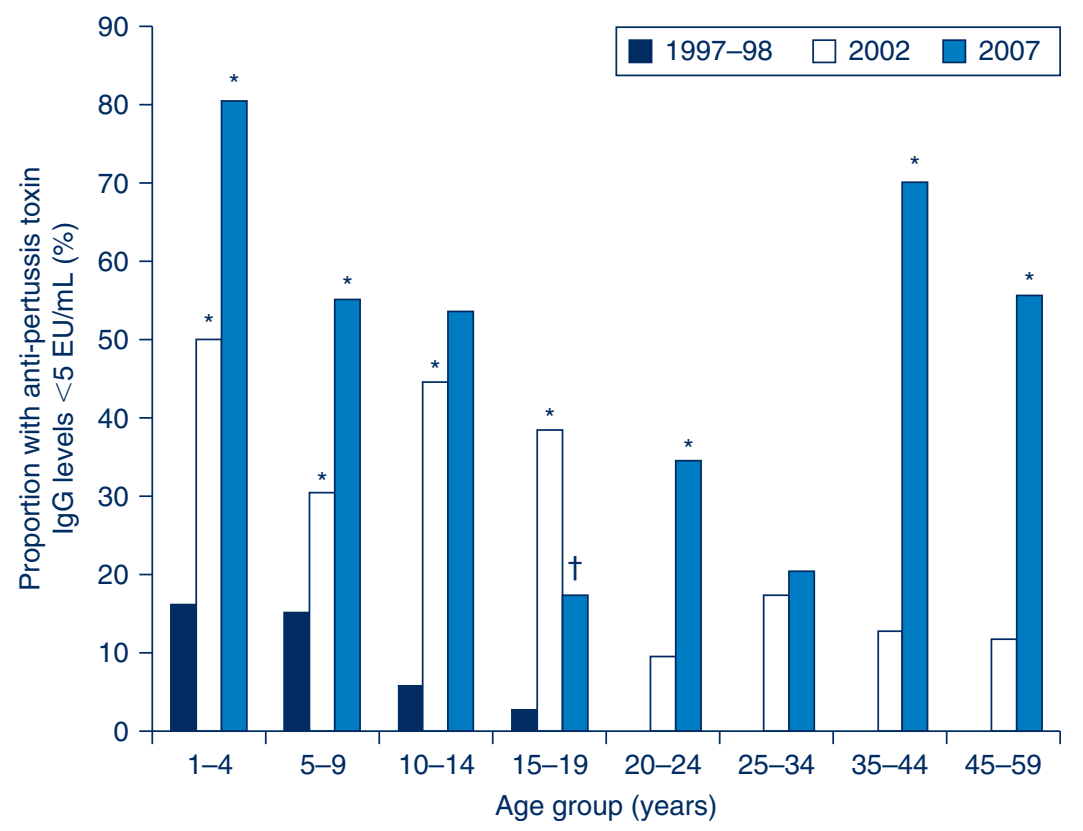

Figure 3. Cross-sectional proportion of undetectable anti-pertussis toxin lgG levels $(<5 \mathrm{EU} / \mathrm{mL})$ in the NSW population in eight age groups (1-4 years through to 45-59 years) in 1997-98, 2002 and 2007.

†Significant decreases compared to the previous collection. *Significant increases compared to the previous collection.

Sources: 1997-98, 2002 and 2007 serosurveys.

\section{Discussion}

This study compares patterns of anti-PT IgG by age, between three time periods. The use of seroepidemiological data for this purpose avoids problems associated with traditional disease surveillance data, such as changes in diagnostic testing practices and physician awareness..$^{10,11}$
Overall, in the 2007 serosurvey there was a decrease in the proportion of high anti-PT IgG levels $(\geq 62.5 \mathrm{EU} / \mathrm{mL})$ across all age groups, compared with earlier serosurveys, which was significant for many age groups. This was in keeping with the relatively low levels of pertussis notifications in this year, which immediately preceded epidemic 
notifications in NSW. ${ }^{1}$ Another unusual finding was the increase in the proportion of undetectable anti-PT IgG levels $(<5 \mathrm{EU} / \mathrm{mL})$, which again was significant in several age groups.

Of particular note is the significant decrease in high antiPT IgG levels in the 1-4-year age group, from $16 \%$ in $1997-98$ to $2 \%$ in 2007 , despite this age group being eligible for recent vaccination, which is thought to be a contributor to high anti-PT IgG levels in serological surveys. $^{2}$ The lower proportion of high anti-PT IgG levels in the 2007 serosurvey in sera from children aged less than 10 years is also reflected in notification data showing low level reporting for this age group in the period 2002-2007. ${ }^{12,13}$ The 1-4-year age group in the 2007 serosurvey were not eligible for an 18-month booster dose of DTPa after it was removed from the schedule in September 2003. ${ }^{7}$ This may have contributed to the significant increase in the proportion of undetectable anti-PT IgG levels in this age group, and is consistent with significant waning of immunity after the primary series of DTPa in the absence of a later booster.

In the 1997-98 serosurvey, adolescents aged 10-14 years (not eligible for a pre-school booster) and 15-19 years (eligible for three-dose primary DTPw vaccination only) had high proportions of high anti-PT IgG levels, which again reflected high notification rates in the 10-19-year age group at this time. ${ }^{14}$ In the 2002 serosurvey, high anti-PT IgG levels continued to be observed for the cohort eligible for a primary series of DTPw only, who were now young adults aged 20-24 years. The relatively high rate of notifications in the 10-19-year age group also continued, reaching a peak in 2001 of 132 notifications per 100000 population. ${ }^{14}$ This rate fell to 62 per 100000 population in 2002, and mirrored the 2002 serosurvey finding of a significantly lower proportion of high anti-PT IgG levels for both the 10-14 and 15-19-year age groups compared to the 1997-98 serosurvey. The decline in anti-PT IgG levels in the 10-14-year age group may be attributable to children who had received the scheduled pre-school booster dose moving into this cohort. ${ }^{15}$

Despite the availability of booster doses during childhood for the now adolescent cohort, coverage was poor, in part due to concerns about the safety of DTPw. ${ }^{10,16}$ When an adult formulated acellular pertussis-containing vaccine (dTpa) became available, it was added to the schedule as a booster dose for adolescents aged 15-17 years. ${ }^{7}$ The dose would target the cohort with sub-optimal childhood booster coverage, who continued to be conspicuous in pertussis notifications ${ }^{17}$ and it was hoped that in the future would prevent an increase in cases due to waning of childhood vaccinations. In 2004, NSW Health delivered this vaccine through a school-based program to those aged 12-19 years and followed this with delivery to Year 7 students (aged $12-13$ years) in 2005. The majority of those targeted in this program were aged 15-19 years at the time of the 2007 serosurvey. This was one of the few age groups to not show a significant decrease in the proportion of anti-PT IgG levels between the 2002 and 2007 serosurveys (Figure 2). Also, in contrast to all other age groups, the 15-19-year age group had a significant decrease in the proportion of undetectable anti-PT IgG levels in 2007, compared to the 2002 serosurvey (Figure 3). This may largely be attributed to the vaccination program, as disease activity between 2002 and 2007 was low. ${ }^{12,13}$

The large increase in undetectable anti-PT IgG levels in the 35-44-year age group is of concern, as this may indicate an increased susceptibility to infection at an age when people are likely to be parents of young infants. Young infants are most at risk from severe disease and death due to pertussis, ${ }^{13,18}$ and parents are an important source of infection. ${ }^{19,20}$ At the present time a 'cocooning strategy' (vaccinating close contacts of infants) is the recommended method of protecting infants too young to be immunised for pertussis. ${ }^{21}$ In March 2009, in response to the epidemic, NSW Health commenced funding of a cocooning dose of dTpa for parents, grandparents and other close contacts of infants less than 12 months of age. ${ }^{22}$ The success of this strategy is currently being investigated.

There were several limitations to this study. The sera used in this study were opportunistically collected rather than randomly sampled. We tried to minimise any biases by obtaining sera that were submitted for a range of diagnostic tests (excluding HIV) to major laboratories throughout NSW that serviced mainly ambulatory populations. We cannot exclude the possibility of selection bias toward a more sick population. No information regarding the clinical status of individuals whose sera were used here was collected and we did not exclude sera that were submitted for the diagnosis of pertussis. However, the method used here has been validated for measles against a prospective, cluster-sampling method and was found to be representative. ${ }^{23}$ The immunisation status of subjects was unknown. While it is possible to estimate immunisation coverage for the childhood population in this study, it is largely unknown for the older age groups.

\section{Conclusion}

The seroepidemiological profiles of the three serosurveys demonstrate fluctuating immunity profiles related to disease patterns and changes in vaccination schedules. The unique seroepidemiological profile from the 2007 serosurvey is interesting and requires further analysis. The small proportion of high anti-PT IgG levels indicative of recent infection, coupled with an increased proportion of very low anti-PT IgG levels, is likely to have set the stage for a large, sustained epidemic. Testing and analysis of serological profiles using a collection from the time of the epidemic would be a valuable means to elucidate the role of population immunity in pertussis epidemic cycles. 


\section{Acknowledgments}

The National Centre for Immunisation Research and Surveillance (NCIRS) is supported by The Australian Government Department of Health and Ageing, the NSW Department of Health and The Children's Hospital at Westmead. Serosurveys conducted by the Centre for Infectious Diseases and Microbiology-Public Health are funded by NCIRS. We wish to acknowledge the staff of the laboratories that provided the sera, Katherine Tudo (Institute of Clinical Pathology and Medical Research) for assistance in the processing and testing of sera and Dr Jodie McVernon (The University of Melbourne) for assistance with the analysis. GlaxoSmithKline provided antigen for the assay. All testing and analysis were conducted at the Centre for Infectious Diseases and Microbiology-Public Health and NCIRS.

\section{References}

1. Spokes PJ, Quinn HE, McAnulty JM. Review of the 2008-2009 pertussis epidemic in NSW: notifications and hospitalisations. N S W Public Health Bull 2010; 21(7-8): 167-73. doi:10.1071/ NB10031

2. de Melker HE, Versteegh FG, Conyn-Van Spaendonck MA, Elvers LH, Berbers GA, van Der Zee A et al. Specificity and sensitivity of high levels of immunoglobulin $\mathrm{G}$ antibodies against pertussis toxin in a single serum sample for diagnosis of infection with Bordetella pertussis. J Clin Microbiol 2000; 38(2): 800-6.

3. Giammanco A, Chiarini A, Maple PA, Andrews N, Pebody R, Gay N et al. European Sero-Epidemiology Network: standardisation of the assay results for pertussis. Vaccine 2003; 22(1): 112-20. doi:10.1016/S0264-410X(03)00514-0

4. Pebody RG, Gay NJ, Giammanco A, Baron S, Schellekens J, Tischer A et al. The seroepidemiology of Bordetella pertussis infection in Western Europe. Epidemiol Infect 2005; 133(1): 159-71. doi:10.1017/S0950268804003012

5. Cagney M, MacIntyre CR, McIntyre P, Puech M, Giammanco A. The seroepidemiology of pertussis in Australia during an epidemic period. Epidemiol Infect 2006; 134(6): 1208-16. doi:10.1017/S095026880600625X

6. Quinn HE, McIntyre PB, Backhouse JL, Gidding HF, Brotherton J, Gilbert GL. The utility of seroepidemiology for tracking trends in pertussis infection. Epidemiol Infect 2010; 138(3): 426-33. doi: $10.1017 /$ S0950268809990707

7. National Health and Medical Research Council. The Australian immunisation handbook. Canberra: Australian Government Department of Health and Ageing; 2003.

8. Gidding H. Australia's national serosurveillance program. NSW Public Health Bull 2003; 14(4-5): 90-3. doi:10.1071/NB03027

9. Giammanco A, Taormina S, Chiarini A, Dardanoni G, Stefanelli P, Salmaso $\mathrm{S}$ et al. Analogous IgG subclass response to pertussis toxin in vaccinated children, healthy or affected by whooping cough. Vaccine 2003; 21(17-18): 1924-31. doi:10.1016/S0264$410 \mathrm{X}(02) 00823-\mathrm{X}$
10. Horby P, MacIntyre CR, McIntyre PB, Gilbert GL, Staff M, Hanlon $\mathrm{M}$ et al. A boarding school outbreak of pertussis in adolescents: value of laboratory diagnostic methods. Epidemiol Infect 2005; 133(2): 229-36. doi:10.1017/S0950268804003401

11. McIntyre P. Lessons from surveillance: solving the pertussis puzzle. N S W Public Health Bull 2003; 14(4-5): 69-71. doi:10.1071/NB03022

12. Brotherton J, Wang H, Schaffer A, Quinn H, Menzies R, Hull B et al. Vaccine preventable diseases and vaccination coverage in Australia, 2003 to 2005. Commun Dis Intell 2007; 31(Suppl): S1-152.

13. Chiu C, Dey A, Wang H, Menzies R, Deeks S, Mahajan D et al. Vaccine preventable diseases in Australia, 2005 to 2007. Commun Dis Intell 2010; 34(Suppl): S1-167.

14. Quinn HE, McIntyre PB. Pertussis epidemiology in Australia over the decade 1995-2005 - trends by region and age group. Commun Dis Intell 2007; 31(2): 205-15.

15. Torvaldsen S, McIntyre PB. Effect of the preschool pertussis booster on national notifications of disease in Australia. Pediatr Infect Dis J 2003; 22(11): 956-9. doi:10.1097/01. inf.0000095198.75170.b6

16. McLennan W. Children's immunisation, Australia. April 1995. ABS Cat. No. 4352.0. Canberra: Australian Bureau of Statistics; 1996.

17. Brotherton J, McAnulty J. A pertussis epidemic in NSW: how epidemiology reflects vaccination policy. NSW Public Health Bull 2003; 14(4-5): 77-81. doi:10.1071/NB03024

18. Cortese MM, Baughman AL, Zhang R, Srivastava PU, Wallace GS. Pertussis hospitalizations among infants in the United States, 1993 to 2004. Pediatrics 2008; 121(3): 484-92. doi:10.1542/peds.2007-1393

19. de Greeff SC, Mooi FR, Westerhof A, Verbakel JM, Peeters JF, Heuvelman CJ et al. Pertussis disease burden in the household: how to protect young infants. Clin Infect Dis 2010; 50(10): 1339-45. doi:10.1086/652281

20. Wendelboe AM, Njamkepo E, Bourillon A, Floret DD, Gaudelus J, Gerber $\mathrm{M}$ et al. Transmission of Bordetella pertussis to young infants. Pediatr Infect Dis J 2007; 26(4): 293-9. doi:10.1097/ 01.inf.0000258699.64164.6d

21. National Health and Medical Research Council. The Australian immunisation handbook. 9th ed. Canberra: Australian Government Department of Health and Ageing; 2008.

22. Della Bosca J. Media release: Whooping cough vaccine not just for kids: 2009. Available from: www.health.nsw.gov.au/news/ 2009/pdf/20090313_02.pdf (Cited 20 May 2011.)

23. Kelly H, Riddell MA, Gidding HF, Nolan T, Gilbert GL. A random cluster survey and a convenience sample give comparable estimates of immunity to vaccine preventable diseases in children of school age in Victoria, Australia. Vaccine 2002; 20(25-26): 3130-6. doi:10.1016/S0264-410X(02)00255-4 